\title{
Fabrication and characterization of a focused ion beam milled lanthanum hexaboride based cold field electron emitter source
}

Gopal Singh, Robert Bücker, Günther Kassier, Miriam Barthelmess, Fengshan Zheng, Vadim Migunov, Maximilian Kruth, Rafal E. Dunin-Borkowski, Stephen T. Purcell, and R. J. Dwayne Miller

Citation: Appl. Phys. Lett. 113, 093101 (2018); doi: 10.1063/1.5039441

View online: https://doi.org/10.1063/1.5039441

View Table of Contents: http://aip.scitation.org/toc/apl/113/9

Published by the American Institute of Physics

\section{Articles you may be interested in}

Energy loss due to defect formation from ${ }^{206} \mathrm{~Pb}$ recoils in SuperCDMS germanium detectors Applied Physics Letters 113, 092101 (2018); 10.1063/1.5041457

Utilizing magnetic field to study the impact of intramolecular charge transfers on the open-circuit voltage of organic solar cells

Applied Physics Letters 113, 093301 (2018); 10.1063/1.5028163

A hexagonal-framed magnetoelectric composite for magnetic vector measurement

Applied Physics Letters 113, 092902 (2018); 10.1063/1.5022094

A broadband fiber-optic nonlinear interferometer

Applied Physics Letters 113, 091103 (2018); 10.1063/1.5048198

Optical fiber-driven low energy electron gun for ultrafast streak diffraction

Applied Physics Letters 113, 133502 (2018); 10.1063/1.5039737

Optical trapping and axial shifting for strongly absorbing particle with single focused TEM 00 Gaussian beam Applied Physics Letters 113, 091101 (2018); 10.1063/1.5044463

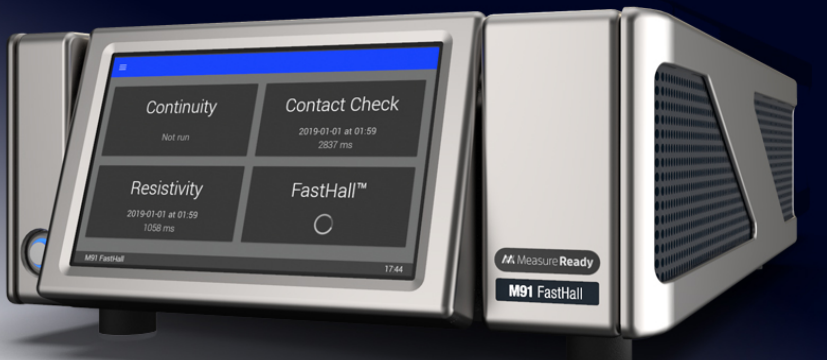

\section{MNM Measure Ready M91 FastHall ${ }^{\mathrm{TM}}$ Controller \\ A revolutionary new instrument for complete Hall analysis}




\title{
Fabrication and characterization of a focused ion beam milled lanthanum hexaboride based cold field electron emitter source
}

\author{
Gopal Singh, ${ }^{1, a)}$ Robert Bücker, ${ }^{1}$ Günther Kassier, ${ }^{1}$ Miriam Barthelmess, ${ }^{2}$ \\ Fengshan Zheng, ${ }^{3}$ Vadim Migunov, ${ }^{3,4}$ Maximilian Kruth, ${ }^{3}$ Rafal E. Dunin-Borkowski, ${ }^{3}$ \\ Stephen T. Purcell, ${ }^{5}$ and R. J. Dwayne Miller ${ }^{1,6}$ \\ ${ }_{1}^{1}$ Max Planck Institute for the Structure and Dynamics of Matter, CFEL (Bld. 99), Luruper Chaussee 149, \\ 22761 Hamburg, Germany \\ ${ }^{2}$ Center for Free Electron Laser Science, DESY, Notkestraße 85, 22607 Hamburg, Germany \\ ${ }^{3}$ Ernst Ruska-Centre for Microscopy and Spectroscopy with Electrons and Peter Grünberg Institute, \\ Forschungszentrum Jülich, 52425 Jülich, Germany \\ ${ }^{4}$ Central Facility for Electron Microscopy, RWTH Aachen University, Ahornstrasse 55, 52074 Aachen, \\ Germany \\ ${ }^{5}$ Institute Lumière Matière, Université Claude Bernard Lyon 1 et CNRS, UMR 530, 69622 Villeurbanne, \\ France \\ ${ }^{6}$ Department of Chemistry and Physics, University of Toronto, Toronto, Ontario M5S 3H6, Canada
}

(Received 8 May 2018; accepted 1 August 2018; published online 27 August 2018)

\begin{abstract}
We report on a method of fabricating lanthanum hexaboride $\left(\mathrm{LaB}_{6}\right)$ cold field emission tips with sub-100-nm apices by using a combination of electrochemical etching and focused ion beam milling. The primary advantage of combining the two methods is rapid fabrication while maintaining reproducibility. The $\mathrm{LaB}_{6}$ tips have low work functions and high mechanical stabilities and are chemically inert to residual gases. Field emission characterization was performed on three tips, with apex sizes of 15,85 , and $80 \mathrm{~nm}$ yielding $10 \mathrm{nA}$ cold field emission currents at $0.76,3.9$, and $3.6 \mathrm{kV}$ extraction potentials, respectively. All three tips showed excellent emission current stability for periods exceeding $30 \mathrm{~min}$ in a $5 \times 10^{-9}$ mbar vacuum. Published by AIP Publishing.

https://doi.org/10.1063/1.5039441
\end{abstract}

A field emission electron source is an essential component in a modern transmission electron microscope (TEM), scanning TEM (STEM), and scanning electron microscope (SEM), as it provides an electron spot size down to the subAngstrom range in a STEM or SEM and excellent phase contrast in a high-resolution TEM. ${ }^{1,2}$ Whereas a Schottky thermally assisted field emission (STFE) source has a high emission current and good long-term stability, a cold field emission source (CFES) has a higher source brightness and a lower energy spread. ${ }^{3}$ CFES technology based on crystalline tungsten (W) has long been implemented ${ }^{4}$ but exhibits poorer emission current stability and higher vacuum sensitivity than STFE devices. These issues are related to the work function and robustness of the tips, as well as chemical inertness to residual gases. ${ }^{5}$ The advent of new material fabrication techniques, such as chemical vapor deposition, has allowed the fabrication of carbon-based devices, including carbon nanotubes, ${ }^{6}$ carbon cones, ${ }^{2}$ and doped silicon carbide $^{7}$ emitters, which have excellent brightness, peak emission current, and stability. However, the disadvantages of these sources include difficulties associated with their axial alignment in W wire mounts, ${ }^{8}$ as well as Coulomb effects following emission from such small tips. ${ }^{9}$ An alternative strategy is to employ compound materials such as lanthanum hexaboride $\left(\mathrm{LaB}_{6}\right),{ }^{10-12}$ whose low work function of $2.7 \mathrm{eV}$, low vapor pressure, ${ }^{13}$ chemical inertness, ${ }^{14}$ and high mechanical robustness potentially make it a superior CFES compared to $\mathrm{W}^{15}$ Although functional $\mathrm{LaB}_{6}$ nano-tip

\footnotetext{
${ }^{\text {a) }}$ Author to whom correspondence should be addressed: gopal.singh@mpsd. mpg.de
}

emitters have been prepared using various methods, ${ }^{12,14,16}$ these generally involve many steps, from fabrication to mounting of the tips, making them time-consuming in production and difficult to use as practical CFES devices. Here, we present a simple, fast, versatile, and robust method for the fabrication of $\mathrm{LaB}_{6}$ nano-emitter devices. We employ a two-step process, involving electrochemical etching and focused ion beam (FIB) milling of single crystal $\mathrm{LaB}_{6}$ rods that are permanently fitted to thermally robust, electrically conducting fixtures, resulting in ready-mounted monolithic $\mathrm{LaB}_{6}$ structures. This method in comparison to others ${ }^{2,3,6,24}$ allows easy and superior control over axial alignment as well as over the tip apex size and shape, thereby ensuring a high degree of performance and reliability. Fabricated $\mathrm{LaB}_{6}$ tips have shown excellent stability in comparison to conventional $\mathrm{W}$ field emitters ${ }^{24}$ and earlier $\mathrm{LaB}_{6}$ monolithic field emitters. ${ }^{12}$ Nondecaying emission current is also observed for $24 \mathrm{~h}$ operation (see supplementary material).

Single crystalline $\mathrm{LaB}_{6}$ rods with diameters of $0.60 \mathrm{~mm}$, lengths of $5 \mathrm{~mm}$, and $\langle 100\rangle$ orientation, so chosen because of their low work function and vapor pressure, ${ }^{10}$ were purchased from APTech. Each $\mathrm{LaB}_{6}$ rod was fitted tightly into a short tube made from tantalum (Ta), protruding by about $2 \mathrm{~mm}$ from one end. The Ta tube was wrapped with $0.25 \mathrm{~mm}$ diameter $\mathrm{W}$ wire, and $\mathrm{W}$ wire leads were laser-welded to the pins of a ceramic mount, as shown schematically in Fig. 1(a). This fixture formed the basis of all subsequent processing and characterization steps. Electrochemical etching of the $\mathrm{LaB}_{6}$ rod was then performed using a method similar to that described by Wang et al., ${ }^{16}$ yielding a tip with an apex of sharpness $1-10 \mu \mathrm{m}$, as shown in Fig. 1(b). 


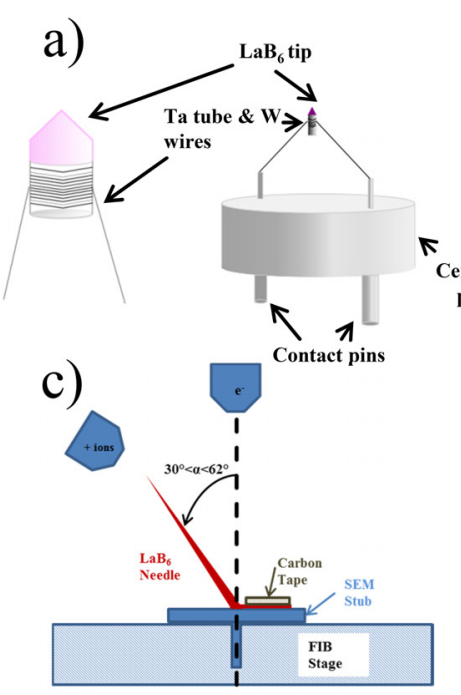

b)

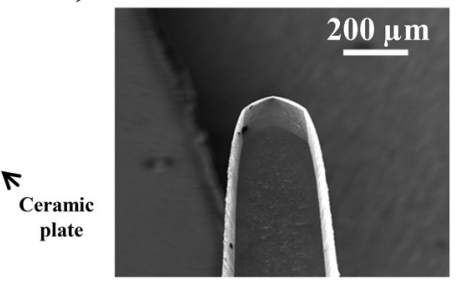

d)

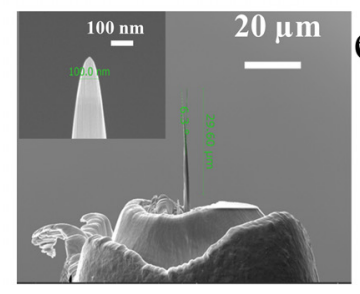

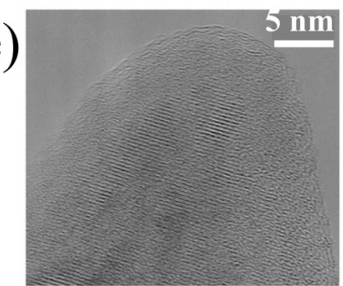

FIG. 1. (a) Schematic diagram of the mounted $\mathrm{LaB}_{6}$ tip protruding outside a Ta tube surrounded by $\mathrm{W}$ wires that are laser welded to contact pins on a ceramic mount; (b) SEM image of an electrochemically etched $\mathrm{LaB}_{6}$ tip of size $1-10 \mu \mathrm{m}$; (c) schematic diagram of FIB milling of a $\mathrm{LaB}_{6}$ tip; (d) SEM image of $\mathrm{LaB}_{6}$ tip after FIB milling. Inset: magnified view of the tip apex; (e) HRTEM image of the tip apex, showing $5 \mathrm{~nm}$ irregularities on the surface.
Further apex size reduction and shaping were carried out using FIB milling, as shown schematically in Fig. 1(c). The rod was aligned on-axis with the ion beam and milled centresymmetrically from the top. This procedure is similar to the annular milling method used for the preparation of atom probe tomography specimens. ${ }^{17}$ Rough milling (with a beam current of $21-60 \mathrm{nA}$ at $30 \mathrm{kV}$ ) was used to sharpen the tip by iteratively removing ring-shaped areas, thereby decreasing the inner diameter. This step was followed by finer milling, involving gradual reduction of the ion current (typically from $2.5 \mathrm{nA}$ to $80 \mathrm{pA}$ ), as well as the outer and inner diameters of the milling pattern. Finally, a polishing step using an ion beam of $5 \mathrm{kV}$ and a current $40 \mathrm{pA}$ for approximately $20 \mathrm{~s}$ with an inner diameter mask setting of zero was applied to reduce the level of gallium implantation. ${ }^{17}$

Figure 1 shows the $\mathrm{LaB}_{6}$ tip morphology subject to the chosen processing steps. An SEM image of the tip after electrochemical etching is shown in Fig. 1(b), revealing an apex size of approximately $10 \mu \mathrm{m}$. Figure $1(\mathrm{~d})$ shows one of the etched tips after FIB milling. This process resulted in conical structures with lengths of approximately $20-30 \mu \mathrm{m}$ and base diameters of $2-5 \mu \mathrm{m}$. The apex diameters were 85,15 , and $80 \mathrm{~nm}$ for tips 1,2 , and 3 , respectively. The combined $\mathrm{LaB}_{6}$ fabrication procedure (electrochemical etching and FIB milling) yielded field emission structures with large bases (tens of $\mu \mathrm{m}$ ) and good mechanical stabilities and electrical conductance. The largely non-selective rate of FIB milling preserves the material stoichiometry and eliminates potential surface contamination and chemical changes caused by electrochemical etching. A high degree of reproducibility is also ensured by this process. ${ }^{18} \mathrm{~A}$ possible concern is destruction of the $\mathrm{LaB}_{6}$ crystallinity due to ion bombardment during FIB milling. HRTEM images of the tip apex recorded in a TEM fitted with a customized sample transfer arm confirmed the presence of damage to the tip in the form of irregularities. A more detailed study of the tip surface is deferred to a future report.

The field emission behaviour of the emitters was then studied. The entire assembly [Fig. 1(a)] was transferred to a field emission characterization chamber with a base pressure of approximately $5 \times 10^{-9} \mathrm{mbar}$, as shown schematically in Fig. 2. A precision high voltage power supply (iseg SHQ 226L) was used to set up the field emission voltage, and the emission current was detected using an ammeter (Keithley 6487) connected to a grounded retractable stainless steel plate positioned at a distance of $30 \mathrm{~mm}$ from the tip. A single microchannel plate (MCP) and phosphor assembly that was lens-coupled to a CCD camera was placed $20 \mathrm{~mm}$ behind the steel plate to image the electron emission pattern. In order to optimize the shape of the tip apex and to achieve stable emission, thermal conditioning was applied..$^{19}$ At temperatures above $800{ }^{\circ} \mathrm{C}$, surface tension forces drive surface migration, rounding, and smoothing of the $\mathrm{LaB}_{6}$ emitter tip. ${ }^{12}$ Finally, in a process known as dulling, the apex radius increases through transport of material from the apex towards the shank. ${ }^{19}$ The temperature was typically fixed in the $900{ }^{\circ} \mathrm{C}-1500^{\circ} \mathrm{C}$ range for approximately $2 \mathrm{~min}$, well below the point at which changes in the surface composition may occur. ${ }^{12}$ Field emission is described by the well-known Fowler-Nordheim (F-N) equation, ${ }^{20}$ which can be written in the simplified form ${ }^{21,22}$

$$
I=A V^{2} \exp \left(-\frac{B}{V}\right)
$$

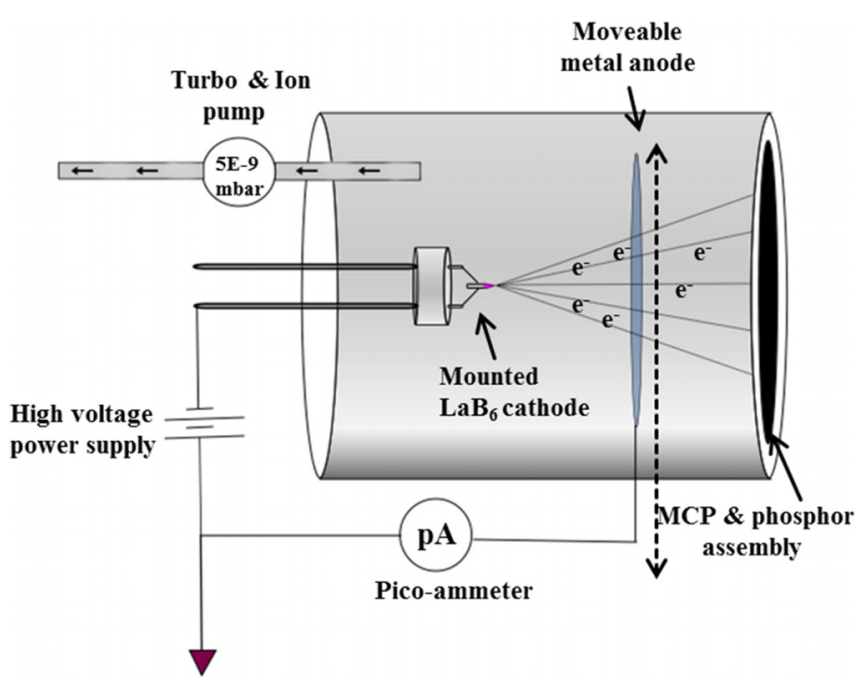

FIG. 2. Schematic diagram of the present field emission characterization setup. 


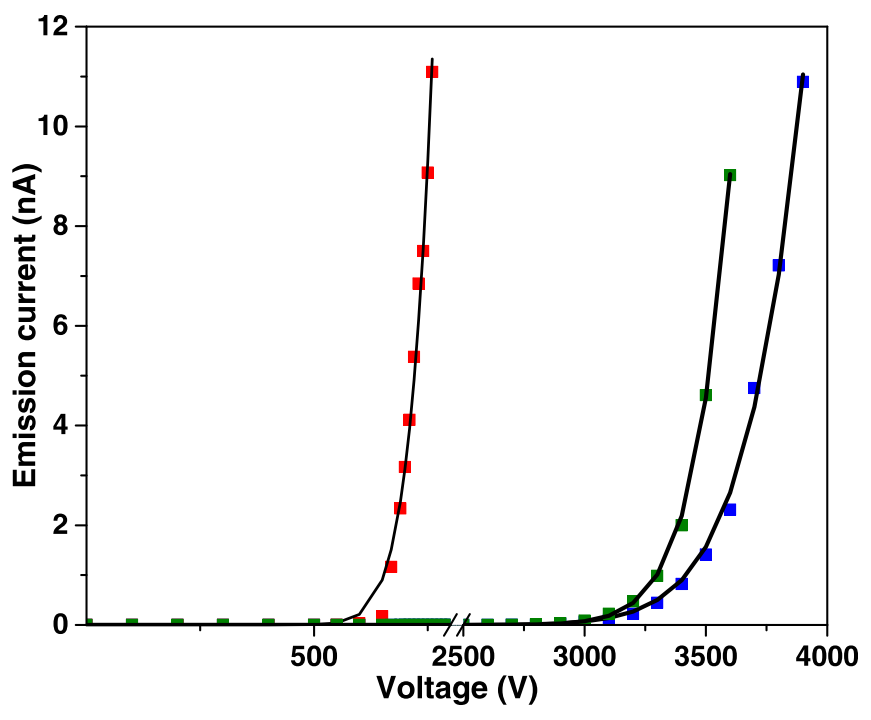

FIG. 3. Current $v s$ voltage $(I-V)$ curves recorded from the $\mathrm{LaB}_{6}$ tips, showing the onset of field emission for tip 1(blue), 2(red), and 3 (green) at $3.0 \mathrm{kV}$, $550 \mathrm{~V}$, and $2.8 \mathrm{kV}$, respectively, confirming the sharpness of tip 2 in comparison with tip 1 and tip 3 .

$$
\begin{aligned}
& A=1.5 \times 10^{-6} \frac{S \beta^{2}}{\phi} \exp (10.4 / \sqrt{\phi}), \\
& B=6.44 \times 10^{9} \phi^{3 / 2} / \beta
\end{aligned}
$$

where $V$ is the applied voltage, $I$ the emission current, $S$ the emitting area, and $\beta$ the field enhancement factor. All of the quantities are given in S.I. units, while the work function $\varphi$ is in eV. From Eq. (1), it is clear that a high emission current can be achieved by choosing a material that has a low work function, as well as by fabricating a sharper tip, as the field enhancement factor is typically inversely proportional to the tip apex radius, and hence, $\beta \propto S^{-1 / 2}$. $I-V$ curves recorded after conditioning are shown in Fig. 3 for all three tips. Least-square fits of Eq. (1) are in excellent agreement with the data for $B_{\mathrm{FN}}^{(1)} \approx 5.9(4) \times 10^{4} \mathrm{~V}, \beta^{(1)} \approx 4.8 \times 10^{5} \mathrm{~m}^{-1}$, for tip $1, B_{\mathrm{FN}}^{(2)} \approx 9.9(5) \times 10^{3} \mathrm{~V}, \beta^{(2)} \approx 2.9 \times 10^{6} \mathrm{~m}^{-1}$ for tip 2 , and $B_{\mathrm{FN}}^{(3)} \approx 5.6(3) \times 10^{4} \mathrm{~V}, \beta^{(3)} \approx 5.1 \times 10^{5} \mathrm{~m}^{-1}$ for tip 3 . The apex radii can be estimated from the empirical relation $\beta \approx 1 / 5 r$ to be $r^{(1)} \approx 410 \mathrm{~nm}, r^{(2)} \approx 70 \mathrm{~nm}$, and $r^{(3)} \approx 395 \mathrm{~nm}$. By using the fit result, the electric field $E$ at the apex of each tip can be estimated to be $1.9 \mathrm{GV} / \mathrm{m}$ at $3.9 \mathrm{kV}$ for tip 1 , $2.2 \mathrm{GV} / \mathrm{m}$ at $760 \mathrm{~V}$ for tip 2 , and $1.8 \mathrm{GV} / \mathrm{m}$ at $3.6 \mathrm{kV}$ for tip 3 . Here, we assumed that the bulk work function of $\mathrm{LaB}_{6}$ is $2.7 \mathrm{eV}$. The effective barrier height subject to this electric field, also known as the Schottky reduced tunneling barrier, ${ }^{23}$ is then estimated to be $1.0 \mathrm{eV}$ for tip $1,0.9 \mathrm{eV}$ for tip 2 , and

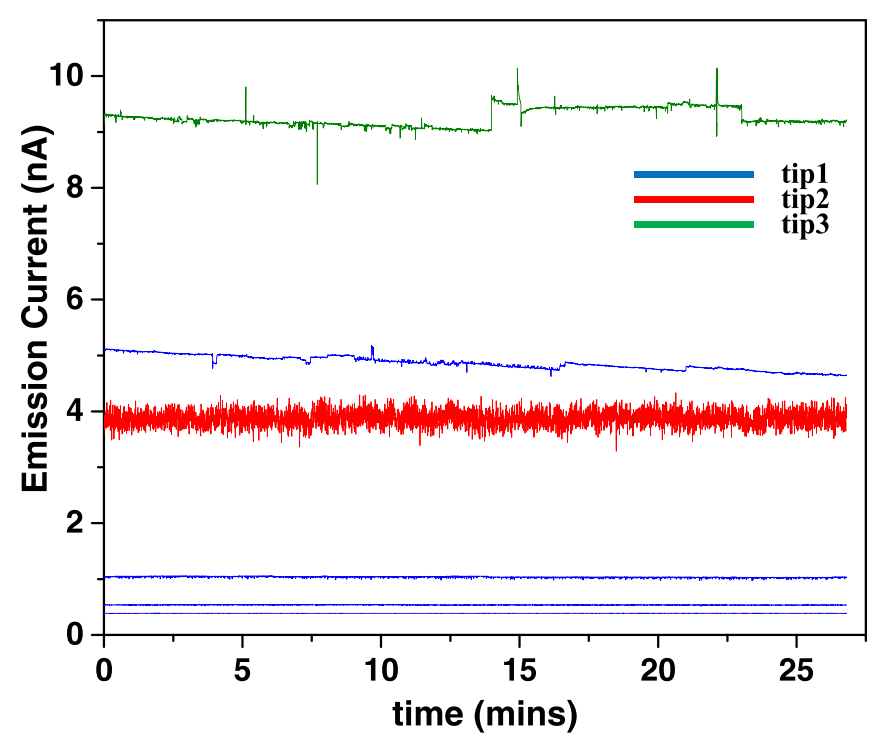

FIG. 5. Temporal stability of the emission current for $\mathrm{LaB}_{6}$ tips. Note the slight jumps for tip 1 and tip 3 .

$1.1 \mathrm{eV}$ for tip 3. The imaging results for the emission pattern are shown in Fig. 4. Gaussian beam spots are suggestive of the Gaussian virtual source. We attribute the distorted appearance of the emission pattern from tip 1 to the imperfect alignment of the tip with respect to the optical axis.

Long-term emission stability is a key factor in the practical utilization of electron emitter devices. In this respect, $\mathrm{LaB}_{6}$ nanowires have been shown to significantly outperform metallic tips. ${ }^{24}$ Figure 5 shows temporal stability profiles of our $\mathrm{LaB}_{6}$ emitters. We observed an almost negligible decay of emission current compared to metallic emitters (typically $\sim 35 \%$ decay over $1 \mathrm{~min}$ ) over $\sim 30 \mathrm{~min}$ and relative RMS noise values of $0.2 \%, 0.6 \%, 1.1 \%$, and $2.5 \%$ (drift-subtracted) for tip 1 at $300 \mathrm{pA}, 500 \mathrm{pA}, 1 \mathrm{nA}$, and $5 \mathrm{nA}$, respectively, $3.5 \%$ for tip 2 at $4 \mathrm{nA}$, and $5 \%$ for tip3 at $9 \mathrm{nA}$. All measurements were performed at a frequency of $3 \mathrm{~Hz}$. Different traces of emission current for tip 2 in comparison to tip 1 and tip 3 are due to the smaller emission area of tip 2 in comparison to tip 1 and tip 3, which results in high fluctuations. ${ }^{26}$ When performing longer stability measurements at currents exceeding approximately $4 \mathrm{nA}$, jumps in current with intermittent plateaus were occasionally observed. These plateaus are attributed to bombardment of the tip with ions desorbed from the anode. ${ }^{25}$

We have presented a method of fabricating $\mathrm{LaB}_{6}$ field emission tips, based on a combination of electrochemical etching, FIB milling, and thermal conditioning. In addition to its simplicity and rapidity of execution, the technique

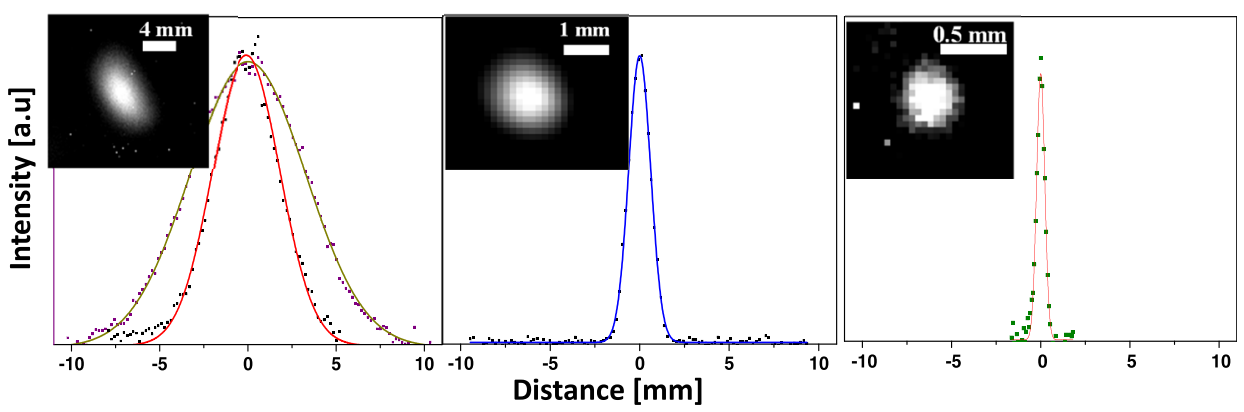

FIG. 4. Field electron micrograph (FEM) images of tip 1 (left), tip 2 (middle), and tip 3 (right) corresponding to emission currents of approximately 10 $\mathrm{nA}$ at $3.9 \mathrm{kV}, 760 \mathrm{~V}$, and $3.6 \mathrm{kV}$, respectively. Gaussian fits (solid line) to the profiles give FWHM values of approximately 7.7 and $4.5 \mathrm{~mm}$ along the long and short axes, respectively, for tip 1 , $1.4 \mathrm{~mm}$ for tip 2 , and $0.4 \mathrm{~mm}$ for tip 3 . 
allows for high reproducibility and customizability in terms of emitter geometry. We characterized the field emission behavior of three tips with different parameters, yielding $10 \mathrm{nA}$ of emission current at $3.9 \mathrm{kV}, 760 \mathrm{~V}$, and $3.6 \mathrm{kV}$. Emission current stability measurements over $30 \mathrm{~min}$ confirmed excellent stability in terms of current drift and noise at a modest vacuum level of approximately $5 \times 10^{-9} \mathrm{mbar}$, suggesting that the chemical inertness of $\mathrm{LaB}_{6}$ is a key advantage over metals for field emission. This property, in combination with the low energy spread expected for a low work function material, makes our emitter a promising candidate for use in electron beam instruments. Further investigations, comprising field ion microscopy, virtual source size measurement, and pulsed emission, will be presented in upcoming articles.

See supplementary material for $24 \mathrm{~h}$ continuous emitter operation (Fig. S1) and the noise spectrum (Fig. S2).

The authors would like to thank Djordje Gitaric from the technical staff and Hendrik Schikora, Martin Kollewe, and Friedjof Tellkamp from the machine physics support group of the Max Planck Institute for the Structure and Dynamics of Matter for help in construction of the field emitter test chamber, laser welding, and construction of the electrochemical etching setup, respectively. This work was funded by the Max Planck Society and the Excellence Cluster "The Hamburg Center for Ultrafast Imaging (CUI) Structure, Dynamics and Control of Matter at the Atomic Scale" of the Deutsche Forschungsgemeinschaft (DFG). Fengshan Zheng and Rafal Dunin-Borkowski acknowledge the European Union for funding through the Marie Curie Initial Training Network SIMDALEE2.
${ }^{1}$ N. de Jonge, Y. Lamy, K. Schoots, and T. H. Oosterkamp, Nature 420, 393 (2002).

${ }^{2}$ F. Houdellier, L. de Knoop, C. Gatel, A. Masseboeuf, S. Mamishin, Y. Taniguchi, M. Delmas, M. Monthioux, M. J. Hÿtch, and E. Snoeck, Ultramicroscopy 151, 107 (2015).

${ }^{3}$ A. H. V. van Veen, C. W. Hagen, J. E. Barth, and P. Kruit, J. Vac. Sci. Technol., B 19, 2038 (2001).

${ }^{4}$ A. V. Crewe, J. Wall, and L. M. Welter, J. Appl. Phys. 39, 5861 (1968).

${ }^{5}$ J. M. Lafferty, J. Appl. Phys. 22, 299 (1951).

${ }^{6}$ N. de Jonge and J.-M. Bonard, Philos. Trans. R. Soc., A 362, 2239 (2004).

${ }^{7}$ M. Choueib, A. Ayari, P. Vincent, M. Bechelany, D. Cornu, and S. T. Purcell, Phys. Rev. B 79, 075421 (2009).

${ }^{8}$ M. Marchand, C. Journet, D. Guillot, J. M. Benoit, B. I. Yakobson, and S. T. Purcell, Nano Lett. 9, 2961 (2009).

${ }^{9}$ P. Kruit, T. Verduin, and B. Cook, in Proceedings of the 2010 8th International Vaccum Electron Sources Conference and Nanocarbon (IVESC) (2010), p. 29

${ }^{10}$ M. A. Uijttewaal, G. A. de Wijs, and R. A. de Groot, J. Phys. Chem. B 110(37), 18459 (2006).

${ }^{11}$ H. Ahmed and A. N. Broers, J. Appl. Phys. 43, 2185 (1972).

${ }^{12}$ M. Futamoto, S. Hosoki, H. Okano, and U. Kawabe, J. Appl. Phys. 48, 3541 (1977).

${ }^{13}$ L. W. Swanson, M. A. Gesley, and P. R. Davis, Surf. Sci. 107, 263 (1981).

${ }^{14}$ H. Zhang, Q. Zhang, J. Tang, and L. C. Qin, J. Am. Chem. Soc. 127(22), 8002 (2005).

${ }^{15}$ M. Futamoto, T. Aita, and U. Kawabe, Mater. Res. Bull. 14, 1329 (1979).

${ }^{16}$ X. Wang, Y. Jiang, Z. Lin, K. Qi, and B. Wang, J. Phys. D: Appl. Phys. 42, 55409 (2009)

${ }^{17}$ M. K. Miller, K. F. Russell, K. Thompson, R. Alvis, and D. J. Larson, Microsc. Microanal. 13, 428 (2007).

${ }^{18}$ N. V. Egorov and E. Sheshin, Field Emission Electronics (Springer, 2017).

${ }^{19}$ P. C. Bettler and F. M. Charbonnier, Phys. Rev. 119, 85 (1960).

${ }^{20}$ R. H. Fowler and L. Nordheim, Proc. R. Soc. A 119, 173 (1928).

${ }^{21}$ T. S. Fisher and D. G. Walker, J. Heat Transfer 124, 954 (2002).

${ }^{22}$ R. G. Forbes, Appl. Phys. Lett. 89, 113122 (2006).

${ }^{23} \mathrm{G}$. Fursey, Field Emission in Vacuum Microelectronics (Kluwer Academic/Plenum Publishers, New York, 2005).

${ }^{24}$ H. Zhang, J. Tang, J. Yuan, Y. Yamauchi, T. T. Suzuki, N. Shinya, K. Nakajima, and L. C. Qin, Nat. Nanotechnol. 11, 273 (2016).

${ }^{25}$ E. E. Martin, J. K. Trolan, and W. P. Dyke, J. Appl. Phys. 31, 782 (1960).

${ }^{26}$ L. W. Swanson and N. A. Martin, J. Appl. Phys. 46, 2029 (1975). 